Vortrag des Medizinhistorikers Prof. Schmiedebach bereitet außerordentliche Mitgliederversammlung der BGPN am 18. März 2018 vor

\section{Psychiatrie, Neurologie und die BGPN in der Zeit des Nationalsozialismus}

Wer die Geschichte der BGPN nachliest, wird darauf stoßen, dass Max de Crinis, der als einflussreichster Nationalsozialist unter den Psychiatern gilt, von 1941-1944 Vorsitzender der Gesellschaft war. Nicht nur deswegen ist die Nervenheilkunde zur Zeit des Nationalsozialismus ein Thema, mit dem sich die BGPN regelmäßig auseinandersetzt. Im Rahmen der Aufarbeitung der Geschichte der BGPN ist auch kritisch angemerkt worden, dass Dr. Hugo Spatz noch als Ehrenmitglied im Mitgliederverzeichnis der BGPN auftaucht.

Spatz war ein deutscher Neuropathologe, der ab 1937 das Kaiser-Wilhelm-Instituts (KWI) für Hirnforschung in BerlinBuch als Direktor leitete. Im Jahr 1938 wurde er Mitglied der NSDAP und gehörte zu den Teilnehmern der Aktion-T4-Besprechung, in der über die Verwertung der Präparate von Euthanasieopfern beraten wurde. Nach dem Zweiten Weltkrieg leitete Spatz unter anderem das Max-Planck-Institut für Hirnforschung in Gießen. Im Jahr 1975 wurde von der deutschen Gesellschaft für Neurologie ein nach ihm benannter Preis ins Leben gerufen. Nach Bekanntwerden der Verwicklung in die Euthanasiemorde wurde der Preis umbenannt.

Auch die BGPN möchte die Ehrenmitgliedschaft von Spatz kritisch überdenken, weshalb sie im Rahmen der Frühjahrstagung am 18.03.2018 zu einer außerordentlichen Mitgliederversammlung einlädt. In Zusammenhang damit sprach Prof. Dr. H.-P. Schmiedebach in der Mittwochsveranstaltung der BGPN am 17.01.2018 über die Nervenheilkunde in der NS-Zeit mit kritischer Beleuchtung der damaligen Akteure. Prof. Schmiedebach ist Arzt, Medizinhistoriker und -ethiker, zu dessen Forschungsschwerpunkten die Medizingeschichte des 18. bis 20. Jahrhunderts und die Geschichte der Psychiatrie gehört.

In seinem Vortrag skizzierte er zunächst das komplexe Wechselverhältnis zwischen Wissenschaft und Politik. Durch die Verschränkung der Humanwissenschaften mit der Politik wurden Techniken gefördert, die auf die Regulierung der biologischen Parameter einer Bevölkerung zielten. Ab ca. 1920 erfolgte eine Veränderung des professionellen Selbstverständnisses mit einer Umorientierung weg vom Patienten zur Administration der Bevölkerungsgesundheit zum Wohl der Volkswirtschaft. Es kam im Verlauf zur Zwangsvereinigung der psychiatrischen und neurologischen Fachgesellschaften mit dem Ziel der Propagation der Rassenhygiene für psychische und neurologische Erkrankungen. Unter maßgeblicher Mitwirkung von de Crinis wurde im Nationalsozialismus das „Euthanasieprogramm" und die neuropathologische Forschung an den Gehirnen ermordeter psychisch und neurologisch Kranker vorangetrieben. Auch die Untersuchungen von Gehirnen durch in Unterdruckversuchen getöteter KZ-Insassen sind belegt.

Hinweise auf eine Mitwirkung von $\mathrm{Hu}$ go Spatz, der 1940 Leiter der "Außenabteilung für Gehirnforschung" des Luftfahrtmedizinischen Forschungsinstituts war, werden durch zwei Briefe Raschers an Himmler und an Romberg („Was ist eigentlich aus unseren schönen Präparaten

\section{Einladung}

Außerordentliche Mitgliederversammlung im Anschluss an die Frühjahrstagung der BGPN am 17.03.2018 um 10.00 Uhr.

Thema der Frühjahrstagung: Spannende Fälle in der Psychiatrie und Neurologie - interaktiv mit TED (kostenfrei!)

\section{Promotionspreis}

15.03.2018 - Bewerbungsschluss Promotionspreis 2018 für herausragende Arbeiten im Bereich der Psychiatrie und Neurologie. Unterlagen an: stephan.koehler@charite.de, Infos unter: www.bgpn.de

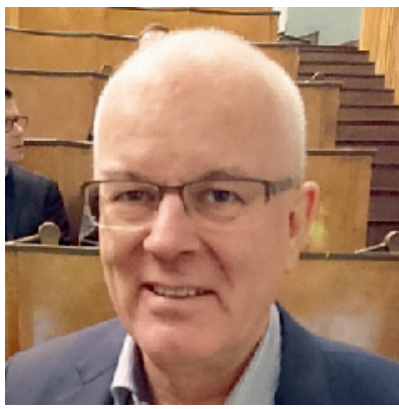

Prof. Dr. Hans-Peter Schmiedebach

bei Spatz geworden?") gegeben. Fast zeitgleich wurde Julius Hallervorden 1938 Leiter der Histopathologischen Abteilung des KWI-Instituts für Hirnforschung BerlinBuch. In dem Institut wurden zwischen 1940 und 1945 etwa 700 Gehirne von ermordeten geistig behinderten und psychisch kranken Menschen, u. a. Kinder aus der Anstalt Brandenburg-Görden unter der Leitung von Hans Heinze, untersucht. Am 19.4.1940 war Hallervorden zusammen mit Hugo Spatz in der Reichskanzlei, wo beide ihr Forschungsverlangen in diesem $\mathrm{Zu}$ sammenhang vorbrachten.

Auch wenn die konkreten Beweise für Spatz' aktive Beteiligung spärlich sind, kann es aus seiner engen Vernetzung im Konglomerat mit de Crinis, Hallervorden, Heinze und der T4-Zentrale als belegt gelten, dass er für seine Forschung an wissentlich unethisch erlangten Hirnpräparaten die Ermordung psychisch oder neurologisch Kranker billigend in Kauf nahm.

Prof. Schmiedebach zog am Ende seines Vortrags das Fazit, dass die Interessen der Gehirnpathologie im Nationalsozialismus die Praxis der Tötungen dominierten. Entscheidung im Sinne einer Wissenschaft in Verantwortung fanden nicht statt, und $\mathrm{Pa}$ tienteninteressen keine Berücksichtigung.

\section{Impressum}

Prof. Dr. Tom Bschor

Redaktion: Dr. Anja M. Bauer

Berliner Gesellschaft für Psychiatrie und Neurologie e. V.

Schlosspark-Klinik, Abteilung für Psychiatrie Heubnerweg 2, 14059 Berlin info@bgpn.de, www.bgpn.de 